\title{
Pyo-Pneumothorax in Patients with Active Pulmonary Tuberculosis: An Analysis of 17 Cases without Intrapleural Fibrinolytic Treatment
}

\author{
Z. Kartaloglu ${ }^{a}$ O. Okutan ${ }^{a} \quad$ T. Işitmangil ${ }^{b} \quad$ E. Kunter ${ }^{a} \quad$ S. Sebit ${ }^{b} \quad$ M. Apaydin ${ }^{a}$ \\ A. $\|^{2}{ }^{a}$ \\ ${ }^{a}$ Department of Pulmonary Diseases and ${ }^{b}$ Thoracic Surgery, Gulhane Military Medical Academia Haydarpasa \\ Training Hospital, Istanbul, Turkey
}

\section{Key Words}

Tuberculosis, pulmonary $\cdot$ Pneumothorax $\cdot$

Pyo-pneumothorax $\cdot$ Empyema

\begin{abstract}
Objective: To review the medical records of patients with active pulmonary tuberculosis (TB) and pyo-pneumothorax (PPT). Subjects and Methods: Medical records of 17 patients (14 male, 3 female, mean age 23.8 years, range 20-52) with PPT and active pulmonary tuberculosis at Gulhane Military Medical Academia Haydarpasa Training Hospital, Istanbul, Turkey, were reviewed from January 1998 to December 2002. The patients were treated with chest tube drainage and chemotherapy. Intrapleural fibrinolytic agents or irrigation was not performed. Results: Pleural fluid samples were available in 14 patients and the mean levels of LDH, protein and glucose in the pleural fluid were 1,767 $\pm 944 \mathrm{U} / \mathrm{l}, 5.2 \pm$ $1.4 \mathrm{~g} / \mathrm{dl}$ and $31.7 \pm 22.6 \mathrm{mg} / \mathrm{dl}$, respectively. Mycobacterium tuberculosis was detected in the pleural effusion of 3 patients. The duration of chest tube drainage was longer in cases who underwent open drainage $(p=0.014)$. At the end of the treatment period 10 patients developed pleural thickening, 4 of them underwent decortication and pneumonectomy was also done in 1 patient. The
\end{abstract}

development of pleural thickening was related to the level of pleural fluid glucose $(p=0.04)$. Conclusion: This study shows that while taking care of patients with pulmonary TB the physician must be aware of the complication of PPT and that adequate chemotherapy and drainage must be duly performed.

Copyright $(2006$ S. Karger AG, Basel

\section{Introduction}

Tuberculosis (TB), one of the oldest known diseases, is still an important community health problem. Although not very often, pneumothorax (Pnx), empyema and pyopneumothorax (PPT) are complications of pulmonary TB and cause significant morbidity and mortality $[1,2]$. Usually, these complications are due to the rupture of a parenchymal nidus or cavity into the pleural space. In most patients with TB, related PPT bronchopleural and/or pleurocutaneous fistulas are common manifestations. These complications make the management even more difficult. Intrapleural fibrinolytic treatment is usually suggested for loculated empyema but there is not enough data to prove that this treatment modality is clearly indicated in all patients with tuberculous empyema and PPT.

\section{KARGER}

Fax +4161306 1234

E-Mail karger@karger.ch

www.karger.com
(C) 2006 S. Karger AG, Basel

$1011-7571 / 06 / 0151-0033 \$ 23.50 / 0$

Accessible online at:

www.karger.com/mpp
Zafer Kartaloglu, Assoc. Prof.

GATA Camlica Chest Diseases Hospital Acibadem/Uskudar

TR-34660 Istanbul (Turkey)

Tel. +90 2163257250, Fax +90 2163257257

E-Mail zkartaloglu@hotmail.com 
In this study we reviewed the records of our patients with active TB and PPT. The aim of the study was to answer the following questions: Is there any specific clinical situation associated with PPT? Why and who develops PPT? Is there any common factor leading to this complication? How could these patients get better medical help?

\section{Subjects and Methods}

Our institution is a referral hospital for TB and is responsible for the care of military personnel in the western part of Turkey. Each year 500-600 new cases are admitted and followed during and after the completion of treatment. We retrospectively reviewed the records of 2,596 patients with the diagnosis of smear-positive pulmonary TB, who were admitted between January 1998 and December 2002.

A total of 17 patients $(0.065 \%)$ with a definite diagnosis of TB with PPT were found. PPT was defined as the situation where Pnx and pleural fluid existed together. All the 17 patients had received standard anti-tuberculosis treatment as described by the World Health Organization [3]. Clinical, radiological and microbiological findings in all these cases were recorded. Routine biochemical studies were performed on the fasting 09:00 a.m. blood samples. Pulmonary TB was radiologically classified as minimal, moderately advanced or far advanced [4]. Minimal lesions included those of slight to moderate density but which did not contain demonstrable cavitation. They may have involved a small part of one or both lungs, but the total extent regardless of distribution did not exceed the volume of lung on one side, present above the second chondrosternal junction and the spine of the fourth or the body of the fifth thoracic vertebra. Moderately advanced lesions were those that were present in one or both lungs, but the total extent did not exceed the following limits: disseminated lesions of slight to moderate density, and these might have extended throughout the total volume of one lung or the equivalent in both lungs; or were dense and confluent lesions limited in extent to one-third the volume of one lung; or had a total diameter of cavitations, if present, of less than $4 \mathrm{~cm}$. Far-advanced lesions were those more extensive than 'moderately advanced'.

The percentage of the collapsed lung in cases with Pnx was calculated by 'The Light Index' [5]. To obtain this index, the cubic power of the mean diameter of collapsed lung is divided by the cubic power of the mean diameter of the hemithorax and the result is subtracted from 1.0 and given as the percentage of Pnx, which was further classified as minimal, partial, subtotal and total $(<20 \%$, $20-40 \%, 41-60 \%$ and $>60 \%$, respectively). Although it is not widely used, we thought that Light Index would be a good and standardized method to classify and describe the degree of Pnx in our cases.

Pleural fluid biochemical (glucose, LDH and total protein) and microbiological examination (smear and culture) results were also evaluated. Initial pleural fluid sampling could not be done in three patients. The diagnosis of empyema was based on the gross visual evaluation of the pleural fluid.

Whenever available, sputum and/or gastric lavage smear and/or culture results were also evaluated. Smears of sputum, pleural biopsy, and pleural fluid were stained with Ziehl-Neelsen and evalu- ated microscopically. Based on the number of the microorganisms on each microscopic field, results were reported as $(-),(+),(++)$, $(+++)$ and $(++++)$. Pleural biopsy specimens were grounded in sterile $0.9 \%$ saline and then inoculated concurrently in LöwensteinJensen and Bactec 12B media. Sputum samples were digested and decontaminated by the $\mathrm{N}$-acetyl- $L$-cysteine-sodium hydroxide method, then concentrated by centrifugation at $3,000 \mathrm{~g}$ for $15 \mathrm{~min}$. Pleural fluid samples were concentrated in the same way. Pleural fluid and sputum sediments were also inoculated on LöwensteinJensen and Bactec 12B. Inoculated media were incubated at $37^{\circ} \mathrm{C}$ up to 6 weeks for Bactec 12B and 8 weeks for Löwenstein-Jensen. Löwenstein-Jensen medium was incubated for an additional 4 weeks if a smear of inoculated material was positive and the culture was negative at 8 weeks. Culture results in Bactec 12B medium were evaluated with appropriate equipment (Bactec 460, Becton Dickinson, Sparks, Md., USA). BACTEC NAP ( $p$-nitro-alpha-acetylamino-beta-hydroxypropiophenone) method was used for the identification of Mycobacterium tuberculosis complex. Indirect radiometric proportion method was used to test the drug sensitivity. Drug concentrations in BACTEC 12B were 0.1, 2.0, 2.0 and $2.5 \mu \mathrm{g} / \mathrm{ml}$ for isoniazid, rifampycin, streptomycin and ethambutol, respectively [6].

Detection of empyema and/or Pnx (partial or more) was the indication for underwater closed tube drainage (UCTD). Open drainage was performed in five cases where prolonged UCTD was not satisfactory. Neither pleural irrigation nor intrapleural fibrinolytic agent treatment had been performed in any cases.

The values were given as mean $\pm \mathrm{SD}$. Multiple linear regression analysis was used to assess the effects of various factors on the length of the time period with UCTD. Logistic regression analysis was used to evaluate the factors affecting pleural thickness. $p<0.05$ was considered statistically significant.

\section{Results}

The study population consisted of 14 male and 3 female patients with the age range of $23.8 \pm 8.0(20-52)$ (table 1). Eleven patients had PPT before admission and 6 were diagnosed with PPT during anti-tuberculous treatment in the hospital. PPT was on the right side in 12 and on the left in 5 cases. One patient presented with a pleurocutaneous fistula.

A chest physician and radiologist did radiological evaluations of the patients separately, and common findings were reported. No major differences were observed between the comments of both specialists. Miliary lesions were observed in 1 and cavitary lesions in 6 patients (fig. 1). Computerized tomography revealed findings suggesting TB and PPT in 7 patients (fig. 2). Other radiological findings are given in table 1 .

Diagnosis of TB was made by sputum culture in 15 , gastric lavage culture in 1, and bronchial lavage culture in 1 of the patients. Sputum culture was positive for AFB in the miliary TB case. Sputum smear examinations for 


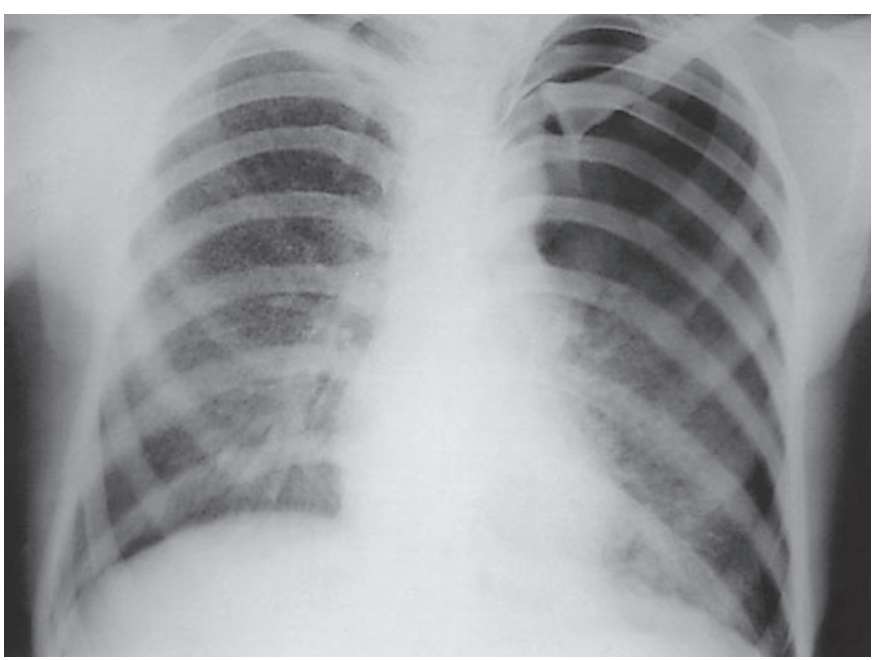

Fig. 1. PA Chest $X$-ray shows the miliary infiltration and PPT on the left side in case 1 .

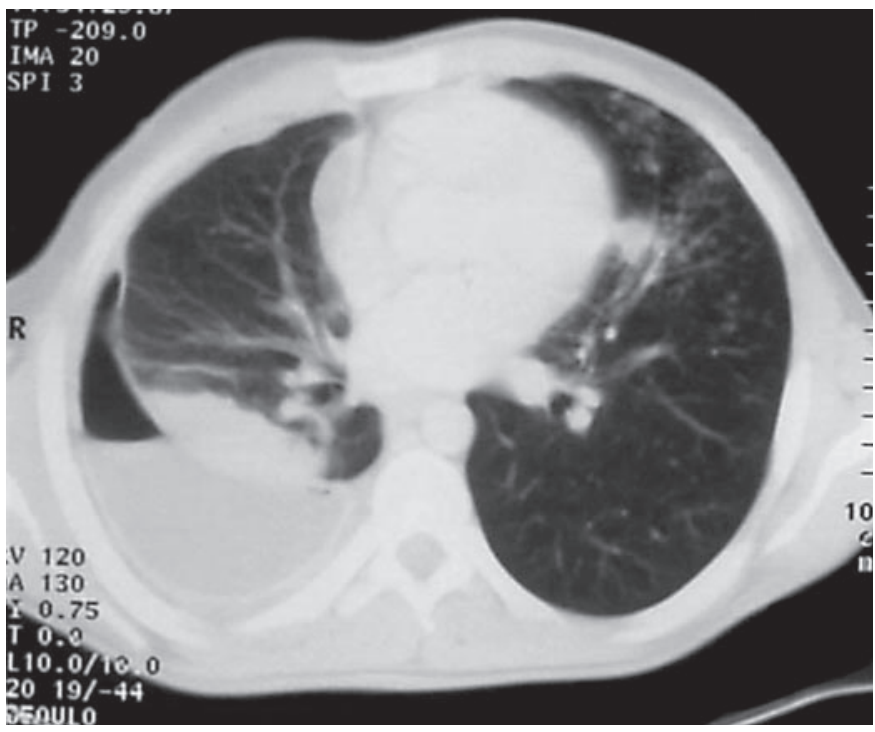

Fig. 2. Thorax CT shows nodular TB infiltration on the left and PPT-associated atelectasis on the right.

Table 1. Clinical, radiological and laboratory features

\begin{tabular}{|c|c|c|c|c|c|c|c|c|c|c|c|c|c|c|}
\hline \multirow{2}{*}{$\begin{array}{l}\text { Case } \\
\text { No. }\end{array}$} & \multirow[t]{2}{*}{ Age } & \multirow[t]{2}{*}{ Sex } & \multirow{2}{*}{$\begin{array}{l}\text { TB } \\
\text { his- } \\
\text { tory }\end{array}$} & \multirow{2}{*}{$\begin{array}{l}\text { Smok- } \\
\text { ing* }\end{array}$} & \multirow{2}{*}{ Complaints } & \multirow{2}{*}{$\begin{array}{l}\text { Chest } \\
\text { X-ray }\end{array}$} & \multirow{2}{*}{$\begin{array}{l}\text { Size } \\
\text { of Pnx. }\end{array}$} & \multicolumn{3}{|c|}{ Pleural fluid } & \multirow{2}{*}{$\begin{array}{l}\text { Total } \\
\text { drainage } \\
\text { time** }\end{array}$} & \multirow{2}{*}{$\begin{array}{l}\text { Open } \\
\text { drainage }\end{array}$} & \multirow{2}{*}{$\begin{array}{l}\text { Residual } \\
\text { pleural } \\
\text { thickness }\end{array}$} & \multirow{2}{*}{$\begin{array}{l}\text { Thora- } \\
\text { cotomy }\end{array}$} \\
\hline & & & & & & & & protein & LDH & glucose & & & & \\
\hline 1 & 21 & M & no & 0 & fever, weight loss, weakness & advanced & subtotal & $(-)$ & $(-)$ & $(-)$ & 5 & no & no & no \\
\hline 2 & 21 & M & no & 10 & chest pain, dyspnea & advanced & subtotal & 6.0 & 2,200 & 37 & 150 & yes & yes & no \\
\hline 3 & 21 & M & no & 6 & cough, weakness, chest pain & advanced & partial & 4.8 & 680 & 17 & 10 & no & yes & no \\
\hline 4 & 24 & M & no & 2 & cough, dyspnea, weight loss & advanced & total & 6.2 & 1,850 & 19 & 150 & no & yes & yes \\
\hline 5 & 25 & $\mathrm{~F}$ & no & 10 & cough, dyspnea, weight loss & advanced & subtotal & 5.9 & 1,950 & 29 & 115 & no & yes & no \\
\hline 6 & 21 & M & no & 5 & dyspnea, chest pain, cough & advanced & partial & $(-)$ & $(-)$ & $(-)$ & 30 & no & no & no \\
\hline 7 & 21 & M & no & 0 & cough, hoarseness, weight loss & advanced & partial & $(-)$ & $(-)$ & $(-)$ & 16 & no & no & no \\
\hline 8 & 22 & M & no & 0 & pleuritic pain, cough, weakness & advanced & partial & 6.2 & 1,890 & 20 & 34 & no & yes & yes \\
\hline 9 & 20 & $\mathrm{~F}$ & yes & 5 & cough, sputum & advanced & subtotal & 4.2 & 540 & 48 & 135 & yes & yes & no \\
\hline 10 & 21 & M & no & 0 & dyspnea & advanced & partial & 7.2 & 3,100 & 25 & 150 & yes & no & no \\
\hline 11 & 52 & $\mathrm{~F}$ & no & 0 & chest pain, weakness & mild & partial & 4.2 & 560 & 35 & 150 & yes & yes & no \\
\hline 12 & 21 & M & no & 5 & cough, chest pain & moderate & total & 2.1 & 1,748 & 7 & 188 & yes & yes & yes \\
\hline 13 & 20 & M & yes & 10 & dyspnea, cough, sputum & moderate & partial & 7.4 & 3,700 & 16 & 90 & no & yes & no \\
\hline 14 & 21 & M & no & 0 & pleuritic pain, cough, fever & moderate & partial & 4.1 & 2,507 & 43 & 30 & no & no & no \\
\hline 15 & 21 & M & no & 0 & dyspnea, cough, sputum & moderate & partial & 5.1 & 1,797 & 43 & 13 & no & no & no \\
\hline 16 & 22 & M & no & 0 & dyspnea, cough, night sweat & advanced & subtotal & 5.2 & 1,500 & 9 & 33 & no & yes & yes \\
\hline 17 & 21 & M & no & 8 & dyspnea, pleuritic pain, cough & moderate & total & 4.4 & 728 & 96 & 13 & no & no & no \\
\hline
\end{tabular}

$*=$ Packs/year; ${ }^{* *}=$ days; $(-)=$ pleural fluids analysis could not be done in cases 1,6 and 7 .

AFB were found to be negative in $5,(+)$ in $1,(++)$ in 6 , $(+++)$ in 2 , and $(++++)$ in 3 patients. Drug resistance was determined in only 1 patient, which was against streptomycin.

Pleural fluid appearance and laboratory analysis results were concordant with empyema in 14 patients. The remaining 3 cases could not be examined in terms of pleural fluid content but the observation of air-fluid levels and gross appearance of the fluid in the chest tube strongly suggested PPT. In 14 patients for whom pleural fluid samples were available, pleural fluid $\mathrm{LDH}$, protein and glucose levels were 1,767.8 $\pm 944.6 \mathrm{u} / \mathrm{dl}(540-3,700), 5.2 \pm$ 

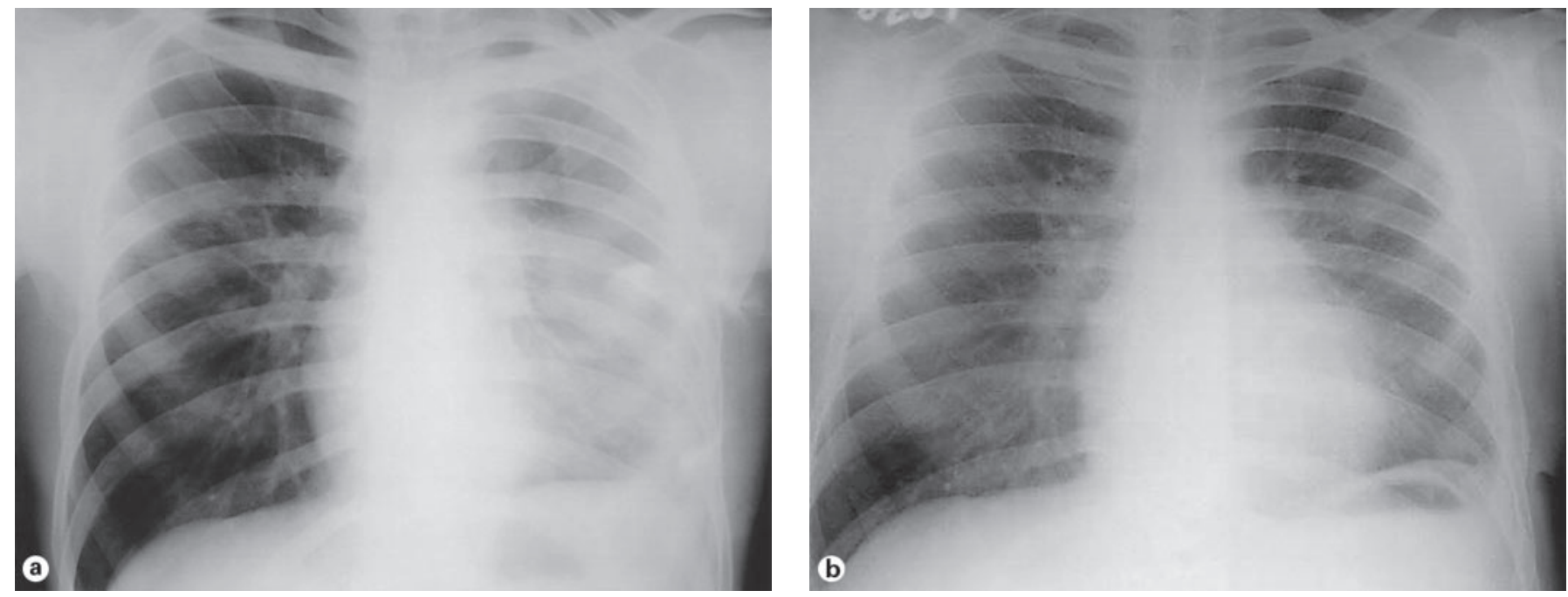

Fig. 3. a PPT resulted in fibrothorax on the left. PA chest X-ray before decortication (case 8). b Chest X-ray shows the radiological appearance of case 8 after decortication. Left costadiafragmatic sinus is minimally occluded.

$1.4 \mathrm{~g} / \mathrm{dl}(2.1-7.4)$, and $31.7 \pm 22.6 \mathrm{mg} / \mathrm{dl}(7-96)$, respectively. M. tuberculosis complex was defined by BACTEC culture in the pleural fluid samples of 3 cases. In the other patients, $M$. tuberculosis could not be shown microbiologically in the pleural fluid. With intermittent sampling, which aimed to prove TB, Acinetobacter calcoaceticus was shown in 2 cases by culture, suggesting superinfection, and appropriate treatment was given.

UCTD was applied to all patients and due to massive air leakage a second UCTD was necessary in 3 patients. Mean duration of total drainage was $77.2 \pm 65.4$ days (5-188) and was more than 10 days in 15 cases. The need for open drainage significantly prolonged the length of the total drainage period $(\mathrm{p}=0.014)$.

After the completion of the medical treatment, all patients were found to be sputum AFB negative. Residual pleural thickness (RPT) was related to level of pleural fluid glucose $(p=0.04)$. The patients with RPT had a mean pleural fluid glucose level of $23.7 \mathrm{mg}$, while those without RPT had a mean level of $51.7 \mathrm{mg}$. Thickening of more than $2 \mathrm{~cm}$ with tomographic evaluation was defined as fibrothorax and an indication for surgical intervention. All 6 patients with fibrothorax were offered surgery, but only 4 underwent decortication (fig. 3). Of these, pneumonectomy was required in one patient whose affected lung could not be expanded due to the destroyed lung. Patients who declined surgery also did not adhere to further hospital visits and tests and could not be followed anymore. No death was reported in this series.

\section{Discussion}

In this series, 17 PPT cases were treated medically and/or with UCTD and/or surgery as appropriate. The clinical course was complicated in most cases with the need for lengthy UCTD application. Pleural fluid which showed a high LDH and protein and low glucose levels was usually associated with RPT. TB is the leading cause of secondary Pnx in high TB prevalence countries and one must consider this point while evaluating any case with Pnx and during the follow-up period of TB [7]. PPT sometimes results in RPT and even destroys the lung. Although requiring further investigation, it has been reported that the pleural fluid content is an important index for the development of RPT [8]. Our findings imply that low pleural fluid glucose level is associated with development of RPT and fibrothorax. Another important issue, from the clinical point of view, is the possibility of low penetration of anti-TB drugs through thickened pleura, which may give rise to insufficient local treatment and drug resistance [9].

In general, the macroscopic and biochemical pleural fluid characteristics of our cases were consistent with empyema. Culture positivity rates for $M$. tuberculosis is usually low in empyema cases. In one series $M$. tuberculosis was culture positive in $60 \%$ of the 35 cases [2]. In our study, $M$. tuberculosis was grown in only 3 cases (17.6\%). However, all of our cases had parenchymal infiltration (miliary, cavitary, fibroproductive, etc.) and we showed 
M. tuberculosis in respiratory secretions of each patient. Since empyema was observed in the first samples of pleural fluid, without a suspicion of interventional contamination, we think that the etiology of PPT is TB in our cases. Extended UCTD duration is sometimes associated with contamination of pleural fluid with organisms other than M. tuberculosis, which can be a major concern. This situation could arise as a complication of diagnostic interventions or extended UCTD.

Application of intrapleural medications is another issue of debate. As of today there are conflicting data regarding the etiopathogenesis of RPT and no treatment option had been proven to be beneficial in its prevention. It has been reported that procedures like irrigation [10] and intrapleural streptokinase application [11] might decrease the risk and degree of RPT. In one study, 26 tuberculous empyema cases that had intrapleural streptokinase treatment were assessed retrospectively and it was shown that 9 of the cases had to be decorticated and 2 of these 9 patients also had pneumonectomy [11]. In another series, where no intrapleural drugs were administered, of the 28 patient with PPT, 2 were decorticated and another 2 had rib resection [12]. In a recent study of 427 cases it was reported that intrapleural streptokinase application had no effect on mortality, need for surgery, and duration of hospitalization, in patients with pleural infection [13]. Our patients were not treated with intrapleural irrigation or intrapleural fibrinolytic drug administration and only 6 of them developed RPT that was severe enough to need decortication, though our results were not different from previous studies whether intrapleural drugs were or were not applied. In fact, empyema or RPT may not be the only factor contributing to severe clinical outcomes resulting in pneumonectomy. Other factors such as impaired host defense, extensiveness of pulmonary TB and disordered underlying lung parenchyma may be important as well. Future studies concerning the prevention of the development of RPT are required and may lead to a decrease in the rate of thoracotomy in these patients. Since there is no convincing evidence that intrapleural drug application has a positive effect on the clinical outcomes, contrary to non-tuberculous cases, we do not use this modality in our patients.

There is also insufficient data to help in identifying patients at higher risk of developing PPT. TB patients with PPT are reported to have more cavitary lesions, which facilitates leakage into the pleural space [14]. Cavitation was reported in 25 (45\%) of 56 patients with pulmonary tuberculosis in the series of Woodring et al. [15]. However, in the current study $35.3 \%$ of the patients $(6 / 17)$ had cavitary lesions and $94 \%(16 / 17)$ of the patients had moderate to far advanced radiological appearance, which suggested that PPT risk was increased with the increased severity of the radiological appearance. Also one of the patients in this PPT group had miliary TB. This is an uncommon finding and, to our knowledge, only 10 miliary TB cases complicated with Pnx have been reported previously [16]. The mechanism that contributes to Pnx in miliary TB has not been described clearly but one idea is that severe coughing episodes and increased alveolar pressure caused by the obliterating effect of miliary nodules might result in alveolar rupture, allowing air to reach the pleural space [17].

It is not surprising that the duration of UCTD treatment is longer in cases with open drainage. Pnx is defined as persistent when it lasts more than 10 days, while treated uninterruptedly by tube thoracostomy with underwater seal, with or without suction [18]. The most common cause of persistence is bronchopleural fistula. In general, if the causative disease is not TB the length of UCTD period is shorter. In a study comparing TB and non-TB diseases as the cause of secondary Pnx, TB patients had a longer hospitalization period and thoracotomy was indicated more frequently [19]. Probably, most of our patients (15/17) with TB-associated PPT also had bronchopleural fistula, and the need for tube thoracostomy with a longer time of treatment. In fact, treatment options are very limited in that kind of patient. Minithoracotomy or decortication via VATS could be other choices, though we did not use these methods in our series.

\section{Conclusion}

Briefly, in active pulmonary TB, PPT is a complication which may occur either before commencement of anti-TB treatment or during treatment. The associated high morbidity and mortality risk warrants that TB-associated PPT be followed carefully and managed with appropriate and well-timed surgical and medical interventions. Also, management without intrapleural fibrinolytic treatment seems to be an effective option. Further studies are needed to establish criteria to recognize patients at higher risk of developing PPT. 


\section{References}

1 Mouroux J, Maalouf J, Padovani B, Rotomondo C, Richelme H: Surgical management of pleuropulmonary tuberculosis. J Thorac Cardiovasc Surg 1996;111:662-670.

-2 Bai KJ, Wu IH, Yu MC, Chiang IH, Chiang CY, Lin TP, Lee YC, Luh KT: Tuberculous empyema. Respirology 1998;3:261-266.

3 World Health Organization: Treatment of Tuberculosis Guidelines for National Programmes, ed 2. WHO/TB/97.220. Geneva, World Health Organization, 1997.

4 Seaton A, Seaton D, Leitch AG: Crofton \& Douglas's Respiratory Diseases, ed 4. Oxford, Blackwell, 1989, pp 409-410.

5 Light RW: Pleural Diseases, ed 2. Philadelphia, Lea \& Febiger, 1990, p 241.

6 Inderlied CB, Salfinger M: Antimicrobial agents and susceptibility test: mycobacteria; in Murray PR (ed): Manual of Clinical Microbiology. Washington, ASM Press, 1995, pp 13851404.

7 Tanaka F, Itoh M, Esaki H, Isobe J, Ueno Y, Inoue R: Secondary spontaneous pneumothorax. Ann Thorac Surg 1993;55:372-376.
$>_{8}$ Kunter E, Ilvan A, Kilic E, Cerrahoglu K, Isitmangil T, Capraz F, Avsar K: The effect of pleural fluid content to the development of pleural thickness. Int J Tuberc Lung Dis 2002; 6:516-522.

9 Iseman MD, Madsen LA: Chronic tuberculosis empyema with bronchopleural fistula resulting in treatment failure and progressive drug resistance. Chest 1991;100:124-127.

10 Ali SM, Siddiqui AA, McLaughlin JS: Open drainage of massive tuberculosis empyema with progressive reexpansion of the lung: an old concept revisited. Ann Thorac Surg 1996; 62:218-223.

11 Al-Kattan KM: Management of tuberculous empyema. Eur J Cardiothorac Surg 2000; 17: 251-254.

12 Hassine E, Marniche K, Bousnina S, Rekhis O, Rabah B, Mustapha MA, Chabbou A, el Gharbi B: Tuberculous pyothorax. 28 cases. Presse Méd 2002;31:921-927.

13 Maskell NA, Davies CWH, Nunn AJ, Hedley EL, Gleeson FV, Miller R, Gabe R, Rees GL, Peto TEA, Woodhead MA, Lane DJ, Darbyshire JH, Davies RLO: U.K. controlled trial of intrapleural streptokinase for pleural infection. N Engl J Med 2005;352:865-874.
14 Yagi T, Yamagishi F, Sasaki Y, Mitutani F, Wada A, Kuroda F: Clinical review of pneumothorax cases complicated with active pulmonary tuberculosis. Kekkaku 2002;77:395399.

15 Woodring JH, Vandiviere HM, Fried AM, Dillon ML, Williams TD, Melvin IG: Update: the radiographic features of pulmonary tuberculosis. AJR 1986;146:497-506.

16 Mert A, Bilir M, Akman C, Ozaras R, Tabak F, Ozturk R, Senturk H, Aktuglu Y: Spontaneous pneumothorax: a rare complication of miliary tuberculosis. Ann Thorac Cardiovasc Surg 2001;7:45-48.

17 Peiken AS, Lamberta F, Seriff NS: Bilateral recurrent pneumothoraces: a rare complication of miliary tuberculosis. Am Rev Respir Dis 1974;110:521-527.

18 Weissberg D, Refaely Y: Pneumothorax: experience with 1,199 patients. Chest 2000;117: 1279-1285.

19 Blanco-Perez J, Bordon J, Pineiro-Amigo L, Roca-Serrano R, Izquierdo R, Abal-Arca J: Pneumothorax in active pulmonary tuberculosis: resurgence of an old complication? Respir Med 1998;92:1269-1273. 